\title{
Management of Hyperglycemia in the Neurosurgery Patient
}

\author{
Rene Daniel;:1,2, Satya Villuri; ${ }^{1}$ Kevin Furlong ${ }^{3}$ \\ 1 Farber Hospitalist Service, Vickie and Jack Farber Institute for Neuroscience, Departments \\ of Neurosurgery and Medicine, Thomas Jefferson University, Philadelphia, PA 19107 \\ 2 Division of Infectious Diseases, Department of Medicine, Thomas Jefferson University, \\ Philadelphia, PA 19107 \\ 3 Division of Endocrinology, Diabetes and Metabolic diseases, Department of Medicine, \\ Thomas Jefferson University, Philadelphia, PA 19107
}

Reprint (with editor's permission): Hosp Pract (1995). 2017 Oct:45(4):150-157. doi:10.1080/2154833 1.2017.1370968. Epub 2017 Aug 30. Management of hyperglycemia in the neurosurgery patient. Daniel R, Villuri S, Furlong K.

*Corresponding author. Address: Rene Daniel, MD, PhD, 909 Walnut St, Clinical Office Building 3rd floor, Philadelphia PA 19197. Tel.: 215-390-0535, email: rene.daniel@jefferson.edu.

\begin{abstract}
Hyperglycemia is associated with adverse outcomes in patients who are candidates for or underwent neurosurgical procedures. Specific concerns and settings that relate to these patients are preoperative glycemic control, intraoperative control, management in the neurological intensive care unit (NICU), and postoperative control. In each of these settings, physicians have to ensure appropriate glycemic control to prevent or minimize adverse events. The glycemic control is usually managed by a neurohospitalist in co-management with the neurosurgery team pre- and postoperatively, and by the neurocritical care team in the setting of NICU. In this review article, we outline current standards of care for neurosurgery patients with diabetes mellitus and/or and hyperglycemia and discuss results of most recent clinical trials. We highlight specific concerns with regards to glycemic controls in these patients including enteral tube feeding and parenteral nutrition, the issues of the transition to the outpatient care, and management of steroid-induced hyperglycemia. We also note lack of evidence in some important areas, and the need for more research addressing these gaps. Where possible, we provide suggestions how to manage these patients when there is no underlying guideline.
\end{abstract}

Keywords: neurosurgical patient, hyperglycemia, hypoglycemia, glycemic control, management, standard of care

\section{INTRODUCTION}

In the general inpatient population, both high blood glucose levels and low glucose levels are associated with unfavorable outcomes. ${ }^{1,2}$ This is also true for neurosurgical patients. Both prospective and retrospective studies showed that hyperglycemia is a risk factor for poor outcomes in traumatic brain injury, and increases both short and long-term mortality in patients with primary intracerebral hemorrhage and wound infections following spinal procedures. ${ }^{3-7}$ It is also associated with increased risk for vasospasm following aneurysmal subarachnoid hemorrhage and poor functional outcome after acute ischemic stroke. 8,9 A retrospective study of 918 patients undergoing craniotomy and spine-related neurosurgical procedures demonstrated that preoperative hyperglycemia [defined as blood glucose (BG) exceeding $120 \mathrm{mg}$ / $\mathrm{dL}$ ] was associated with increased risk of postoperative complications at all levels of care. ${ }^{10}$ Similarly, hyperglycemia in diabetes patients (defined as blood BG over 200 $\mathrm{mg} / \mathrm{dL}$ ) was an independent predictor of morbidity after brain biopsy in a retrospective study. ${ }^{11}$

Surgical procedures themselves are known to induce intraoperative hyperglycemia. ${ }^{12}$ On the other hand, anesthetics moderate glucose and glucose metabolism. ${ }^{13,14}$ For example, volatile anesthetics inhibit secretion of insulin in response to glucose, and thus inhibit the stressinduced hyperglycemia. ${ }^{13}$ Clinical and animal studies suggest that hyperglycemia exacerbates neurological damage due to brain ischemia. ${ }^{15,16}$ These results, in addition to those outlined above, emphasize the deleterious effects of poor glycemic control.

Increase in complications due to hyperglycemia among neurosurgical patients has also been reported for systems other than the central nervous system (CNS): genitourinary (acute renal injury and urinary tract infections), digestive (paralytic ileus), the peripheral nervous system (PNS, peripheral nerve root lesions) and cardiovascular (cardiac arrhythmias). ${ }^{17,19}$ Electrolyte abnormalities were also associated with hyperglycemia in neurosurgical patients. ${ }^{17,19}$

Finally, another factor that often induces hyperglycemia in the neurosurgery patients is the use of glucocorticoids Risk factors for steroid induced hyperglycemia include traditional risk factors for type 2 diabetes mellitus, along with dose and duration of steroid usage. ${ }^{20}$ Other risk factors are concurrent immunosuppression, hypomagnesemia and hepatitis $C$ virus infection. ${ }^{20}$ Glucocorticoids can induce hyperglycemia in non-diabetics and worsen glucose control in established diabetic patients, with negative consequences outlined above.

Similar to hyperglycemia, hypoglycemia was also associated with complications in neurosurgical patients, including increased mortality. ${ }^{18}$ This is due to the crucial role of glucose for the brain metabolism. ${ }^{21}$ In addition, the glucose 
metabolism in brain in controlled by glucose transfer, and the glucose levels in blood thus may not reflect the glucose levels in brain cells, which could be lower than expected based on blood glucose levels. ${ }^{22}$ The case thus can be made for careful glycemic control at any point of management of a neurosurgical patient.

In this article, we review glycemic goals and management practices for patients, which were admitted for a neurosurgical procedure. For practical purposes, we have subdivided the management into six steps. Each step has its own specific features and concerns. We will proceed chronologically, starting with admission, preoperative management, continuing with intraoperative glycemic control, postoperative care in intensive care unit setting and on the floor, and finishing with discharge and transition into outpatient care.

\section{ADMISSION}

The neurosurgical procedures can be divided into elective and emergent. Depending on the procedure, patients can be admitted on the day of surgery, or if need be, prior to surgery (for example, if extensive surgery is anticipated and patient needs pre-operative optimization under close supervision). The management of BG in emergent procedures is described below in the section 4 (Intraoperative control). In this section, we concentrate on elective and thus scheduled procedures.
Prior to admission, the patient will likely undergo preoperative testing at an outpatient clinic. At present, there is no consensus with the respect to the glycemic goal of patients with a planned neurosurgical procedure. A review article suggests that the goal should be consistent with the goals as outlined for outpatient population by then American Diabetes Association ${ }^{1,23}$ According to the latest ADA guideline, the pre-meal glucose targets are 80-130 $\mathrm{mg} / \mathrm{dL}$ and hemoglobin $\mathrm{A} 1 \mathrm{C}$ levels less than $7 \%$. The $A 1 C$ goal can be relaxed to $8 \%$ in selected patients \{e.g. patients with history of severe hypoglycemic episodes, and/or limited life expectancy, see ADA guideline for details. ${ }^{1,23}$ In the absence of evidence to the contrary, we suggest that these targets are likely appropriate for patients planning to have a neurosurgical procedure. ${ }^{1}$ Hemoglobin A1C level should be checked during the preoperative testing visit unless there is one available within last 3 months. ${ }^{1,24}$ Admission orders should clearly state whether the patient is a Type 1, Type 2 or a non-diabetic.

\section{PREOPERATIVE GLYCEMIC CONTROL}

Prior to admission, patients with diabetes mellitus type 2 are likely treated with either oral diabetic medications or with insulin. Oral medications may have unpredictable pharmacokinetics and/or pharmacodynamics during surgery, and are known to have a number of undesirable side effects. ${ }^{25}$ For these reasons, we suggest oral medications should be held the day of surgery. ${ }^{25}$ This is consistent with the general ADA recommendations [1]. Given the half-life of metformin, some authors suggest holding it 24 hours prior to surgery, and 48-72 hours is suggested for sulfonylureas. ${ }^{23}$ However, there is no recent clinical evidence suggesting better outcomes with these strategies. ${ }^{23}$

For insulin-dependent diabetes mellitus type 2, many studies suggest to continue the full dose of the long acting insulin (e.g. glargine)..$^{1,23}$ However, a recent observational study in type 2 diabetic patients undergoing ambulatory surgery showed that the optimal dosing of glargine is $60-87 \%$ of the daily dose, which is our recommendation. ${ }^{26}$

Some type 2 diabetics are on premixed insulin (long and short acting components). In this situation, there appear to be two options: if the surgery is not scheduled for a very near future, we suggest that patient can be transited, in collaboration with their PCP or endocrinologist, to a regimen including long acting and meal insulin. The meal insulin then can be held prior to surgery. This appears to be a preferable solution, since premixed insulin regimen on the floor results in more hypoglycemic episodes than a regimen including long acting and meal insulin. ${ }^{27}$ Alternatively, ADA guidelines recommend that the patient can take $1 / 2$ the dose of the premixed

\section{Table 1. Preoperative Adjustment of Diabetic Medications}

\begin{tabular}{|c|c|c|c|}
\hline Medication & DM Type 1 & DM Type 2 & Reference \\
\hline \multicolumn{4}{|l|}{$\operatorname{Re}$} \\
\hline Oral & - & Stop & 25 \\
\hline Basal insulin (long acting, one dose daily) & Full dose & Decrease to $60-87 \%$ dose & 12,26 \\
\hline Basal Insulin (split dose) & - & $\begin{array}{l}\text { Full dose the night, decrease as } \\
\text { above in am }\end{array}$ & 24 \\
\hline Meal insulin & Stop & Stop & 11 \\
\hline Mixed Isulin & - & $1 / 2$ intermediate component in am & 1 \\
\hline Correction dose ( $q 6 h$ ) & Start & $\begin{array}{c}\text { Start } \\
26\end{array}$ & 1,12 \\
\hline Insulin pump & Continue* & - & $26-30$ \\
\hline
\end{tabular}

*Stop pump and start insulin infusion if emergent or a long procedure 
insulin dose, which corresponds to the long acting component. For example, if a patient is taking 50 units of $70 / 30$ before breakfast, $70 \%$ of this dose is conceptually intermediate acting insulin and would be 35 units. One could give $1 / 2$ (17 units) of that amount in the form of $\mathrm{NPH}$ or longer acting insulin the AM of surgery. ${ }^{1}$

Type 1 diabetics should receive their full dose of basal insulin preoperatively. A common mistake is to stop basal insulin when a patient is made NPO. This could lead to rapidly elevating BG that could precipitate DKA. Type 1 diabetics thus should continue their basal insulin. ${ }^{12}$

Insulin pumps are becoming a more popular insulin delivery method in both type 1 and type 2 diabetics. In general, insulin pumps can be left in place for shorter elective procedures. They have been shown to be safe in several studies ${ }^{28-32}$ If the patient demonstrates decompensated metabolic control, the patient should be started on an insulin infusion and the pump be removed. In general, the infusion should be in place for $1 / 2$ hour before the pump is discontinued. It is optimal to have the patient test their basal rates before surgery. Patients should also change their insertion site and reservoir the day before surgery and bring extra pump supplies with them.

Post-operatively, the pump can be continued as long as the patient is awake, alert and oriented and is capable of managing the pump. If the patient is not capable of appropriate self- management, the pump should be discontinued after a subcutaneous basal/bolus regimen is started.

Insulin pumps should be discontinued for emergent or longer neurosurgical procedures.

Finally, if a patient is NPO following admission and waits for the procedure for an extended period of time, correction dose (sliding scale) insulin should be added to the long-acting insulin regimen (see above), with point of care glucose checks every 6 hours. ${ }^{1,12}$ ADA guidelines propose the inpatient glycemic goals to be $140-180 \mathrm{mg} / \mathrm{dL} .{ }^{1}$ There is no evidence suggesting that these goals should be different for neurosurgical patients. Therefore, we think these goals are appropriate in this patient population.

The proposed adjustments in insulin regimen for patients with insulin-dependent diabetes mellitus are summarized in Table 1.

\section{INTRAOPERATIVE CONTROL}

Recent results (see above in Introduction) suggest that close and careful glucose control during a procedure is critical for a successful outcome. ${ }^{13-16} \mathrm{~A}$ very recent observational study showed that severe intraoperative hyperglycemia (defined as $B G>180 \mathrm{mg} / \mathrm{dL}$ ) is associated with postoperative composite infections after craniotomy. ${ }^{33}$

These results suggest that the intraoperative glycemic goals should be BG less than $180 \mathrm{mg} / \mathrm{dL}$ for neurosurgical procedures. In the absence of direct evidence, we suggest that the lower end of the range should be glucose no lower than $140 \mathrm{mg} / \mathrm{dL}$, which is the target of ADA for hospitalized patients. ${ }^{1}$ We note that a retrospective study on patients undergoing a cardiac surgery suggested that blood BG less or equal to $140 \mathrm{mg} / \mathrm{dL}$ was associated with worse outcomes. ${ }^{34}$

Oral glucose-lowering agents are to be stopped prior to procedure (see above). However, the last time when they were taken should be taken into consideration. Assuming their effect wore off, the glucose levels during the procedure are dependent on the intrinsic control by the patient's body, and on insulin doses given during the procedure. Point of care glucose checks can be used to monitor BG during procedure. For patients who were only on oral medications at home, correction insulin doses appear to be an acceptable approach.

There are two options for BG control in patients who already are treated with insulin at home: long acting insulin together with the correction dose insulin, and continuous intravenous insulin infusion. There is no evidence suggesting which of these methods leads to better outcomes in neurosurgical patients.

Table 2. Management of BG in patient receiving enteral tube feeding (TF) or parenteral nutrition.

\begin{tabular}{|c|c|c|c|}
\hline Situation & Basal Insulin & Bolus Insulin & Comments \\
\hline Continuous TF & $\begin{array}{l}\text { Continuous IV insulin infusion until patient } \\
\text { reaches goal TF. This will be TDD* of insulin } \\
\text { for that TF rate. } \\
\text { Basal insulin will be } 30-40 \% \text { of the TDD* in the } \\
\text { form of glargine daily or NPH/detemir BID }\end{array}$ & $\begin{array}{l}\text { Bolus: Regular insulin q } 6 \text { hours with } \\
\text { regular insulin correction. }\end{array}$ & $\begin{array}{l}\text { Patients with DM1 always require basal } \\
\text { insulin. } \\
\text { ADA recommends basal insulin plus rapid } \\
\text { acting correctional insulin every } 4 \text { hours. }\end{array}$ \\
\hline Nocturnal TF & $\mathrm{NPH}$ and regular insulin given before TF & $\begin{array}{l}\text { Can use correction dose rapid acting } \\
\text { insulin during TF }\end{array}$ & $\begin{array}{l}\text { Give an AM dose of NPH as well in insulin } \\
\text { requiring DM2, SM1 and patients on gluco- } \\
\text { corticoids }\end{array}$ \\
\hline Bolus TF & Add regular insulin to TPN bottle. & Rapid acting insulin with the TF bolus & \\
\hline Parenteral & Add regular insulin to TPN bottle. & Rapid acting correction dose insulin & \\
\hline Interrupted enteral & $\begin{array}{l}\text { Run D5 or D10 if insulin was given. } \\
\text { Reduce basal dose. }\end{array}$ & Hold bolus doses & Continue basal insulin for DM1 \\
\hline
\end{tabular}


Multiple factors could be involved in selection of a method to control BG. These include the type of diabetes and the length of procedure. Given all the factors that could influence BG levels, we suggest that the continuous insulin infusion is a better option for the type I diabetics undergoing a long procedure and prevents strong fluctuations in the BG levels. The same applies to the type II diabetics that have a highly insulindependent diabetes mellitus and had a poor BG control prior to procedure. For non-insulin requiring type 2 diabetics, long acting insulin with correction dose insulin seems to be an appropriate option. For emergency procedures in patients with hyperglycemia with or without type 2 diabetes, intravenous insulin infusion seems to be an appropriate option, since it can enable better control if unexpected variations in $B G$ levels occur.

\section{POSTOPERATIVE GLYCEMIC CONTROL IN THE NEURO- LOGICAL INTENSIVE CARE UNIT SETTING}

Following a procedure, neurosurgical patients often spend a significant period of time in the neurological intensive care unit (NICU). Given the stress-induced hyperglycemia, hyperglycemia associated with $\mathrm{SAH}, \mathrm{ICIH}$ and $\mathrm{TBI}$, and the effect on anesthetics on glucose control, it is not surprising that hyperglycemia is common among critically ill patients. ${ }^{35,36}$ What should be the glycemic goal in this setting? A meta-analysis of sixteen randomized clinical trials by Kramer et al. analyzed protocols that used tight glycemic targets (70-140 mg/dL) versus protocols that kept the glucose levels below 144-300 mg/dL. ${ }^{35}$ A few conclusions were drawn. First, tight glucose control resulted in better neurological outcomes, but had no effect on mortality. However, hypoglycemic episodes were far more common than in the tightly controlled group. Second, although the "loose control" protocols were associated with worse neurological outcomes, these were observed only when glucose levels were above 200 $\mathrm{mg} / \mathrm{dL}$. The outcomes for the range between 140-180 mg/dL were not worse than in the tight glucose control group. Similar results were obtained by Ooi et al, who performed a meta-analysis of outcomes of tight glucose control versus conventional glucose control in critically ill neurological and neurosurgical patients. ${ }^{37}$ Nine studies were included in the analysis, five of which were restricted to neurosurgical patients and four included neurological patients. The results showed that tight glucose control improved the neurological outcomes and reduced rates of infection. Again, mortality was not affected by the tight glucose control, but it did result in more hypoglycemic events. These results did not enable the authors to determine the optimal glucose targets, which means the question of appropriate glycemic targets remains, to a certain degree open. ${ }^{37}$ Nevertheless, the available evidence suggest that the glycemic goal between 140-180 mg/dL appears to be appropriate for critically ill neurosurgical patients. ${ }^{35-37}$

Critically ill patients immediately postprocedure are either managed with intravenous insulin infusion or longacting insulin with correction dose insulin. Nutrition is usually restarted as early as possible post-procedure. Patients can be transitioned from intravenous insulin infusions to subcutaneous regimens when they are clinically stable. It is imperative to remember that there should be an overlap of 1-2 hours between the first subcutaneous insulin dose and the discontinuation of the insulin infusion. ${ }^{23}$

A significant percentage of critically ill patients require enteral tube feeding, either due to being intubated or to dysphagia. In these cases, there is a paucity of data regarding the optimal method of glucose management with enteral nutrition. We follow the ADA guidelines with some modifications depending on the situation, ${ }^{1,23,38}$ (Table 2). If a patient is capable of oral intake, he or she can be then restarted on a long acting and meal insulin. We note that the doses of the subcutaneous insulin should be calculated from the insulin does that were given during the insulin infusion, using standard protocols. Oral home diabetic medications are not restarted the critical care setting. Instead, these patients are treated with an insulin-based regimen, as outlined above.

Per the ADA guidelines, glucose monitoring for patients who can eat should be performed before meals. ${ }^{1}$ In the patient who do not eat, glucose monitoring is advised every 4-6 h. ${ }^{24}$ If patients are managed with intravenous insulin infusion, BG testing is should occur in more frequent intervals, from every $30 \mathrm{~min}$ to every 2 h. ${ }^{1}$

\section{POSTOPERATIVE GLYCEMIC CONTROL ON THE FLOOR}

BG levels are usually managed in our practice and in many neurosurgical practices after transition from NICU or directly from the postoperative unit by a team of neurohospitalist and the neurosurgical primary team.

Observational studies in general surgery patients have concluded that perioperative hyperglycemia is associated with poor outcomes such as delayed wound healing, increased chances of infection, prolonged length of stay and increased health care costs. ${ }^{39-41}$

ADA guidelines recommend for general inpatient population the BG goal 140-180 $\mathrm{mg} / \mathrm{dL}$, and in the absence of contrary evidence, this appears to be an appropriate goal for neurosurgical patients on general floor. ${ }^{1}$ When $B G$ falls below this goal, therapeutic regimen should be adjusted. However, for patients who have history of successfully maintaining tight glycemic control in the outpatient setting and are clinically stable, the glycemic goal can be lower than 140 $\mathrm{mg} / \mathrm{dL} .{ }^{1}$ The glycemic goal is usually achieved by a combination of basal and bolus insulin. ${ }^{1} \mathrm{~A}$ randomized clinical trial of insulin therapy in the management of general surgical patients demonstrated the superiority of basal-bolus insulin when compared to the correction dose insulin in achieving glycemic goals. ${ }^{39}$ One caveat of the trial is that the BG goal was 100-140 mg/dL, which is lower than the BG goal recommended above.

Finally, a recent trial suggests that sitagliptin, either alone or in combination with basal insulin, was as efficient in achieving BG goals as a basal-bolus insulin regimen. ${ }^{42}$ However, more studies will be needed to compare efficacy and safety of sitagliptin-based regimen with the standard therapy. ${ }^{1,42}$

As noted in Introduction, glucocorticoids are commonly used in the neurosurgical patient. They pose a significant challenge to the achievement of glucose goals. 
Steroids induce mainly post-prandial hyperglycemia, with peaks occurring usually in the afternoon and evening. ${ }^{43-45}$ Checking a baseline fasting BG or hemoglobin A1C prior to the procedure helps predict patients at risk for developing hyperglycemia while on steroids. ${ }^{20}$

Several approaches have been proposed for the treatment of glucocorticoidinduced hyperglycemia, but no published studies have investigated the efficacy of these approaches. Our suggestions are as follows. Bedside glucose testing should be initiated for patients with or without a history of diabetes. Another important factor is a carbohydrate consistent diabetic diet. Insulin therapy should be initiated for patients whose blood sugars exceed established glucose goals. Patients already on basal-bolus therapy will need increases in their insulin doses, particularly in the prandial component. Occasionally, patients will require a continuous IV insulin infusion for severe and persistent elevations in BG despite subcutaneous insulin ${ }^{24}$

It is very important that insulin dosing be adjusted during glucocorticoid tapers. Insulin should be proactively adjusted to avoid hypoglycemia.

\section{GLYCEMIC CONTROL DURING TRANSITION TO OUTPATIENT CARE}

The discharge is the next critical step in managing neurosurgery patients and their BG levels. While there are clinical studies for management of inpatient hyperglycemia (see above), little is known about management of hyperglycemia in neurosurgical patients after discharge.

The admission A1C can be helpful in formulating an appropriate treatment regimen. A recent discharge algorithm for glycemic medication adjustment based on admission $\mathrm{A} 1 \mathrm{C}$ found the average $\mathrm{A} 1 \mathrm{C}$ reduction decreased from $8.7 \%$ on admission to $7.3 \%$ three months after discharge. ${ }^{46}$

In the case of patients who do not continue steroids as outpatients, it is usually possible to discharge them on their home diabetic medications. ${ }^{1}$ Patients who were not well controlled prior to admission will require necessary

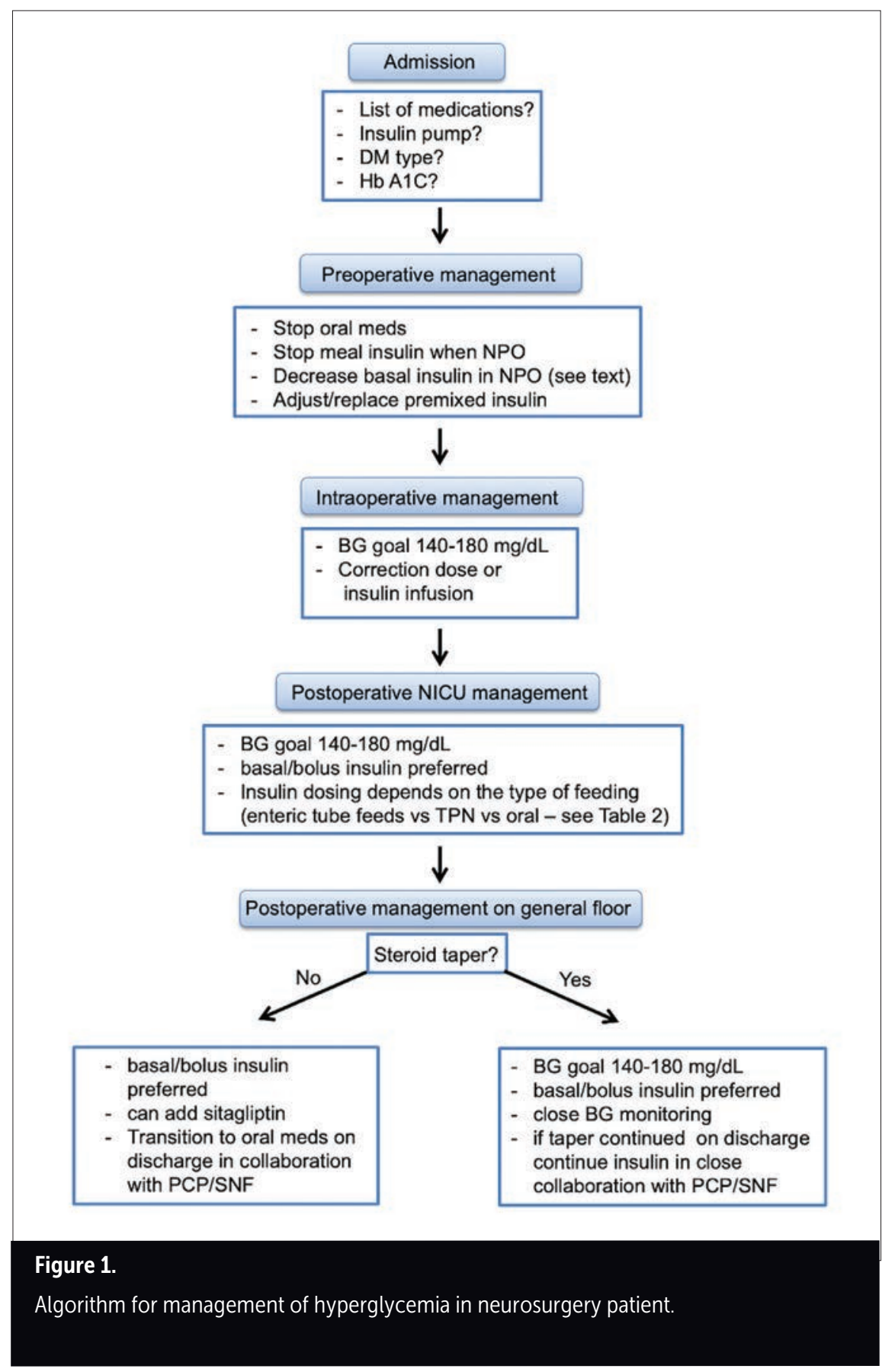

adjustments to their regimen to optimize glucose control. This often necessitates the utilization of insulin. Insulin naïve patients need training in self-injection technique and diabetes education. This includes the prevention and correction of hypoglycemic episodes. The insulin requirements are based on the inpatient insulin requirements. PCP should be informed of the changes in the patient's medications.

If patients continue to be treated with glucocorticoids upon discharge, BG management becomes a complex process. Elderly patients and debilitated neurosurgical patients are even more 
challenging when it comes to selfadjusting insulin doses at home. There is no "standard steroid taper" and different neurosurgery practitioners use different steroid tapers depending on the clinical scenario. Hence, as of now there is no standard protocol for management of hyperglycemia at home while on steroid taper. We thus believe that it is critical that the patient monitors their BG before meals and at bedtime. The must also be in contact with the health care provider who is managing their glucose levels.

A survey of patients discharged on insulin that included a high proportion of patients on glucocorticoids showed that $49 \%$ of patients reported BG level over $300 \mathrm{mg} / \mathrm{dL} .{ }^{47}$ A survey done in a diabetic center at Massachusetts General Hospital between 2011 and 2013, showed the incidence of hypoglycemia ( $B G<60$ $\mathrm{mg} / \mathrm{dl}$ ), among a diabetic population discharged on glucocorticoids was up to $46 \% .{ }^{48}$ Given the risks of hyper- and hypoglycemia, development of standard protocols for management of $B G$ in patients on steroid taper outside of hospital setting is highly desirable.

If blood sugars are below $70 \mathrm{mg} / \mathrm{dl}$, patients in our practice are advised to reduce insulin dose by $20 \%$. If blood sugars are above $300 \mathrm{mg} / \mathrm{dl}$, the insulin dose should be increased by $20 \%$. These are arbitrary adjustments and need to be tapered according to the dose of steroids and BG levels at home, in conjunction with PCP. Given the lack of supporting studies, we categorize these suggestions and those below as expert opinion-level recommendations.

Taken together, we suggest that close follow-up with PCP, ideally within 1 week is essential. For newly diagnosed diabetics while in the hospital or steroid induced hyperglycemia requiring insulin, the PCP should be sent a copy of the hospitalization record or a brief discharge summary including a list of the discharge medications. The neurohospitalist should contact the PCP and convey the information to make sure they are aware for the need for a close follow-up.

\section{SUMMARY AND DISCUSSION}

In this article, we review management of hyperglycemia in the neurosurgical patient. Neurosurgery patients pass through several levels of care, necessitating careful adjustment in the management of their hyperglycemia. These include the preparation for the neurosurgical procedure (including the NPO regimen), intraoperative management, postoperative management (including possible stays in neurological intensive care units), and finally the transition to outpatient care. Our suggested approach to the management of BG levels in neurosurgery patients is outlined in the Figure 1.

\section{Several major points bear repeating:}

1. Outpatient oral anti-hyperglycemia medication should be held prior to the procedure.

2. For insulin-requiring type 2 diabetics, basal long-acting insulin should be taken at a decreased dose and the meal insulin held prior to procedure (see above). If the patient is being treated with premixed insulin, the long-acting component of the premixed insulin should be taken at a decreased dose (see above).

3. Insulin pumps do not have to be discontinued prior to a short procedure. If the BG control decompensates, the patient undergoes a longer or an emergent procedure, or is unable to control the insulin pump, the pump should be stopped and replaced by an insulin infusion during the procedure.

4. The available evidence suggests that a relaxed blood $\mathrm{BG}$ control, with a glycemic goal of $140 \mathrm{mg} / \mathrm{dL}$ to 180 $\mathrm{mg} / \mathrm{dL}$ leads to better outcomes for neurosurgery patients than a tight BG control with a goal less than $140 \mathrm{mg} / \mathrm{dL}$. This result is consistent with the BG management goals proposed in the ADA guideline for general inpatient population.

5. Given the significant role that enteric tube feedings play in the management of neurosurgery patients, the attending physician needs to be aware of the BG management methods.

6. After transition out of the neurological intensive care unit, glycemic goals are usually achieved by treatment with insulin. The combination of a basal insulin (e.g. glargine) and bolus insulin (meal and correction dose) yields significantly better outcomes that management with the correction doses only, or management with premixed insulin. ${ }^{27,39}$ However, very recent evidence suggests that addition of sitagliptin leads to an improved glucose control. ${ }^{42}$

7. Use of steroids significantly impacts BG management. Patients often require higher doses of insulin, particularly in the prandial component. BG levels need to be followed closely, particularly with decreasing glucocorticoid dosages to prevent hypoglycemia.

8. Neurosurgery patients discharged to home or to a skilled nursing facility (SNF) need close follow up in order to maintain appropriate glycemic goals. The transition of care plays a crucial role at discharge. An early appointment with the primary care physician is a must. Interaction between patient's primary care physician and inpatient attending physician is necessary to ensure smooth transition of care, and appropriate glycemic control. If the patient is being discharged to an intermediate care facility, it is advisable that the attending physician prepares detailed instructions and contacts the facility physician to ensure the appropriate glycemic control.

The summary which is listed above and our suggested management guidelines are based on differing levels of evidence. There is a new clinical study, which supports the decrease of basal insulin (glargine) prior to procedure. ${ }^{26}$ On the other hand, outside of the ADA guideline, we did not identify any studies that would support that management of the premixed insulin as suggested in our review, although it appears to be somewhat consistent with the management of long-acting basal insulin. We rely here on the expert opinion. The Rabbit 2 Surgery trial, which compares management of hyperglycemia with basal vs correction dose only insulin, was a double blinded, randomized trial and thus provides a high level of confidence to the proposal that basal insulin should be included in the management of inpatient neurosurgery 
patients. ${ }^{39}$ Additionally, premixed insulin should be avoided in the inpatient setting. There are multiple studies that support the relaxed insulin control in the neurological intensive unit (see above). In addition, there is an ongoing clinical trial addressing intensive versus non-intensive insulin therapy for hyperglycemia after traumatic brain injury, which may shed more light on this issue. ${ }^{49}$

An important and developing issue is whether preadmission diabetes mellitus should impact glycemic goals in hospitalized patients. A growing body of literature suggests that in general ICU patients, while tight glucose control is associated with decreased mortality in the nondiabetic population, diabetics may benefit from higher glucose goals. ${ }^{50,51}$ Similar, a very recent observational study reported that chronic pre-morbid hyperglycemia increase the risk of hypoglycemia and modifies the association between acute hypoglycemia and mortality. ${ }^{52}$ It remains to be established whether these conclusions can be extended to neurocritical patients. If so, the glycemic goals will have to differ for patients with preadmission diabetes and nondiabetics. We hope this issue will be addressed in near future by researchers in the field.

Unfortunately, there is no evidencebased algorithm for the management of the steroid taper. Here, we again rely on the expert opinion. There is, however, an ongoing clinical trial addressing the treatment of inpatient diabetes on steroids. Unfortunately, no results are available at the time of this publication. ${ }^{53}$

A crucial issue with regards to glycemic control is the development and management of hypoglycemia in neurosurgical patients. In this article, we have concentrated on the management of hyperglycemia, and did not approach systematically the issue of hypoglycemia, We note that hypoglycemia issue in general inpatient population has been addressed in the ADA guideline. ${ }^{1}$ Nevertheless, hypoglycemia in neurosurgical patients is a topic that deserves an extensive analysis and warrants a different article.

Taken together, there is need for more evidence addressing management of hyperglycemia in neurosurgery patients. Existing gaps in our knowledge include glycemic goals in the presence vs. absence of preexisting diabetes mellitus, evidence-based protocols for management of steroid taper, and use of oral diabetic medications in inpatient setting. Nevertheless, the current level of knowledge allows us to propose an approach to the hyperglycemia management that we hope neurohospitalists find helpful and informative (Figure 1). We also hope that our review will stimulate research in the areas that need high quality of evidence to improve outcomes of neurosurgery patients.

\section{ACKNOWLEDGEMENTS}

We are grateful to the Department of Neurosurgery and Farber Hospitalist Service leadership and administrative staff for support in preparation of this article.

\section{Statement of Conflict of Interest}

The authors declare no competing interests.

\section{REFERENCES}

1. ADA, Standards of medical care in diabetes 2016. Diabetes Care, 2016. 39(Supplement 1): p. S99-S104.

2. Clement, S., et al., Management of diabetes and hyperglycemia in hospitals. Diabetes Care, 2004. 27(2): p. 553-91.

3. Shi, J., et al., Review: Traumatic brain injury and hyperglycemia, a potentially modifiable risk factor. Oncotarget, 2016. 7(43): p. 7105271061.

4. Salim, A., et al., Persistent hyperglycemia in severe traumatic brain injury: an independent predictor of outcome. Am Surg, 2009. 75(1): p. 25-9.

5. Guo, X., et al., Hyperglycemia and Mortality Risk in Patients with Primary Intracerebral Hemorrhage: A Meta-Analysis. Mol Neurobiol 2016. 53(4): p. 2269-75

6. Olsen, M.A., et al., Risk factors for surgical site infection following orthopaedic spinal operations. J Bone Joint Surg Am, 2008. 90(1): p. 62-9.

7. Wang, T., et al., Factors predicting surgical site infection after posterior lumbar surgery: A multicenter retrospective study. Medicine (Baltimore), 2017. 96(5): p. e6042.

8. Inagawa, T., K. Yahara, and N. Ohbayashi, Risk factors associated with cerebral vasospasm following aneurysmal subarachnoid hemorrhage. Neurol Med Chir (Tokyo), 2014. 54(6): p. 465-73.

9. Luitse, M.J., et al., Chronic hyperglycemia is related to poor functional outcome after acute ischemic stroke. Int J Stroke, 2017. 12(2): p. 180-186.
10. Davis, M.C., et al., Preoperative hyperglycemia and complication risk following neurosurgical intervention: A study of 918 consecutive cases. Surg Neurol Int, 2012. 3: p. 49.

11. McGirt, M.J., et al., Independent predictors of morbidity after image-guided stereotactic brain biopsy: a risk assessment of 270 cases. J Neurosurg, 2005. 102(5): p. 897-901.

12. $12 . \quad$ Sudhakaran, S. and S.R. Surani, Guidelines for Perioperative Management of the Diabetic Patient. Surg Res Pract, 2015. 2015: p. 284063

13. 13. Tanaka, T., H. Nabatame, and Y. Tanifuji, Insulin secretion and glucose utilization are impaired under general anesthesia with sevoflurane as well as isoflurane in a concentration-independent manner. J Anesth, 2005. 19(4): p. 277-81.

14. Tanaka, K. and Y.M. Tsutsumi, [Glucose Metabolism: Stress Hyperglycemia and Glucose Control]. Masui, 2016. 65(5): p. 495-502.

15. Sieber, F.E., The neurologic implications of diabetic hyperglycemia during surgical procedures at increased risk for brain ischemia. $J$ Clin Anesth, 1997. 9(4): p. 334-40.

16. Pasternak, J.J., et al., Hyperglycemia in patients undergoing cerebral aneurysm surgery: its association with long-term gross neurologic and neuropsychological function. Mayo Clin Proc, 2008. 83(4): p. 406-17.

17. Bilotta, F., et al., Glycemia management in neurocritical care patients: a review. $J$ Neurosurg Anesthesiol, 2009. 21(1): p. 2-9.

18. Bilotta, F. and G. Rosa, Glucose management in the neurosurgical patient: are we yet any closer? Curr Opin Anaesthesiol, 2010. 23(5): p. 539-43.

19. Kavanagh, B.P. and K.C. McCowen, Clinical practice. Glycemic control in the ICU. N Engl J Med, 2010. 363(26): p. 2540-6.

20. Hwang, J.L. and R.E. Weiss, Steroid-induced diabetes: a clinical and molecular approach to understanding and treatment. Diabetes Metab Res Rev, 2014. 30(2): p. 96-102.

21. Mergenthaler, P., et al., Sugar for the brain: the role of glucose in physiological and pathological brain function. Trends Neurosci, 2013. 36(10): p. 587-97.

22. Morea, V., et al., Glucose transportation in the brain and its impairment in Huntington disease: one more shade of the energetic metabolism failure? Amino Acids, 2017.

23. Godoy, D.A., et al., Perioperative glucose control in neurosurgical patients. Anesthesiol Res Pract, 2012. 2012: p. 690362.

24. Moghissi, E.S., et al., American Association of Clinical Endocrinologists and American Diabetes Association consensus statement on inpatient glycemic control. Diabetes Care, 2009. 32(6): p. 1119-31.

25. Kokil, G.R., et al., Pharmacology and chemistry of diabetes mellitus and antidiabetic drugs: a critical review. Curr Med Chem, 2010. 17(35): p. 4405-23. 
26. Demma, L.J., et al., Effect of basal insulin dosage on blood glucose concentration in ambulatory surgery patients with type 2 diabetes. J Clin Anesth, 2017. 36: p. 184-188.

27. Bellido, V., et al., Comparison of BasalBolus and Premixed Insulin Regimens in Hospitalized Patients With Type 2 Diabetes. Diabetes Care, 2015. 38(12): p. 2211-6.

28. Sobel, S.I., et al., Safety and Efficacy of a PeriOperative Protocol for Patients with Diabetes Treated with Continuous Subcutaneous Insulin Infusion Who Are Admitted for Same-Day Surgery. Endocr Pract, 2015. 21(11): p. $1269-76$

29. Thompson, B.M., et al., Perioperative Management of Patients with Diabetes and Hyperglycemia Undergoing Elective Surgery. Curr Diab Rep, 2016. 16(1): p. 2

30. Mackey, P.A., et al., Update on a Quality Initiative to Standardize Perioperative Care for Continuous Subcutaneous Insulin Infusion Therapy. J Diabetes Sci Technol, 2015. 9(6): p. 1299-306

31. Boyle, M.E., et al., Insulin pump therapy in the perioperative period: a review of care after implementation of institutional guidelines. J Diabetes Sci Technol, 2012. 6(5): p. 1016-21.

32. Corney, S.M., et al., Comparison of insulin pump therapy (continuous subcutaneous insulin infusion) to alternative methods for perioperative glycemic management in patients with planned postoperative admissions. J Diabetes Sci Technol, 2012. 6(5): p. 1003-15.

33. Gruenbaum, S.E., et al., Severe Intraoperative Hyperglycemia Is Independently Associated With Postoperative Composite Infection After Craniotomy: An Observational Study. Anesth Analg, 2017

34. Duncan, A.E., et al., Role of intraoperative and postoperative blood glucose concentrations in predicting outcomes after cardiac surgery. Anesthesiology, 2010. 112(4): p. 860-71.
35. Kramer, A.H., D.J. Roberts, and D.A. Zygun, Optimal glycemic control in neurocritical care patients: a systematic review and metaanalysis. Crit Care, 2012. 16(5): p. R203.

36. Bilotta, F. and G. Rosa, Optimal glycemic control in neurocritical care patients. Crit Care, 2012. 16(5): p. 163.

37. Ooi, Y.C., et al., Tight glycemic control reduces infection and improves neurological outcome in critically ill neurosurgical and neurological patients. Neurosurgery, 2012. 71(3): p. 692-702; discussion 702

38. Mabrey, M.E., et al., Managing hyperglycemia and diabetes in patients receiving enteral feedings: A health system approach. Hosp Pract (1995), 2015. 43(2): p. 74-8.

39. Umpierrez, G.E., et al., Randomized study of basal-bolus insulin therapy in the inpatient management of patients with type 2 diabetes undergoing general surgery (RABBIT 2 surgery). Diabetes Care, 2011. 34(2): p. 256-61.

40. Pomposelli, J.J., et al., Early postoperative glucose control predicts nosocomial infection rate in diabetic patients. JPEN J Parenter Enteral Nutr, 1998. 22(2): p. 77-81.

41. Noordzij, P.G., et al., Increased preoperative glucose levels are associated with perioperative mortality in patients undergoing noncardiac, nonvascular surgery. Eur J Endocrinol, 2007. 156(1): p. 137-42

42. Umpierrez, G.E., et al., Safety and efficacy of sitagliptin therapy for the inpatient management of general medicine and surgery patients with type 2 diabetes: a pilot, randomized, controlled study. Diabetes Care, 2013 36(11): p. 3430-5.

43. Oyer, D.S., A. Shah, and S. Bettenhausen, How to manage steroid diabetes in the patient with cancer. J Support Oncol, 2006. 4(9): p. 479-83.
44. Clore, J.N. and L. Thurby-Hay, Glucocorticoid-induced hyperglycemia Endocr Pract, 2009. 15(5): p. 469-74

45. Burt, M.G., et al., Continuous monitoring of circadian glycemic patterns in patients receiving prednisolone for COPD. J Clin Endocrinol Metab, 2011. 96(6): p. 1789-96.

46. Umpierrez, G.E., et al., Hospital discharge algorithm based on admission $\mathrm{HbAlc}$ for the management of patients with type 2 diabetes. Diabetes Care, 2014. 37(11): p. 2934-9.

47. Kimmel, B., M.M. Sullivan, and R.J. Rushakoff, Survey on transition from inpatient to outpatient for patients on insulin: what really goes on at home? Endocr Pract, 2010. 16(5): p. 785-91.

48. Wei, N.J., D.M. Nathan, and D.J. Wexler, Glycemic control after hospital discharge in insulin-treated type 2 diabetes: a randomized pilot study of daily remote glucose monitoring. Endocr Pract, 2015. 21(2): p. 115-21.

49. https://clinicaltrials.gov/ct2/show/NCT0216 1055?term =hyperglycemia+traumatic +brain grank=1

50. Krinsley, J.S., et al., Diabetic status and the relation of the three domains of glycemic control to mortality in critically ill patients: an international multicenter cohort study. Crit Care, 2013. 17(2): p. R37

51. Lanspa, M.J., et al., Moderate glucose control is associated with increased mortality compared with tight glucose control in critically ill patients without diabetes. Chest, 2013 143(5): p. 1226-1234

52. Egi, M., et al., Pre-morbid glycemic control modifies the interaction between acute hypoglycemia and mortality. Intensive Care Med, 2016. 42(4): p. 562-571.

53. https://clinicaltrials.gov/ct2/show/NCT019702 41?term=hyperglycemia+steroids+inpatient+ diabetes\&rank=1 